\title{
Effects of pressure impulse and peak pressure of a shockwave on microjet velocity in a microchannel
}

\author{
Keisuke Hayasaka $^{1}$ Akihito Kiyama ${ }^{1}$ Yoshiyuki Tagawa $^{1}$
}

Received: 12 June 2017 / Accepted: 10 October 2017 / Published online: 25 October 2017

(C) The Author(s) 2017. This article is an open access publication

\begin{abstract}
We experimentally investigate the effect of a shockwave on the velocity of a microjet and underwater cavitation onset in a microchannel, focusing on the pressure impulse and peak pressure of the shockwave. The pressure impulse and peak pressure of the shockwave are separately varied by using non-spherical laser-induced shockwaves (Tagawa et al. in J Fluid Mech 808:5-18, 2016). We conduct two kinds of experiments. In one experiment, we install a narrow tube and a hydrophone in different configurations in large water-filled tank. We measure the shockwave pressure and the jet velocity simultaneously in order to elucidate the effects of pressure profile of shockwaves on the jet velocity. In the other experiment, we record the jet formation and cavitation onset to investigate the effect of cavitation onset on the jet velocity in an L-shaped microchannel. The experimental results suggest that the jet velocity depends only on the pressure impulse of the shockwave, while the probability of cavitation onset depends only on the peak pressure of the shockwave. The above findings provide new insights for advancing a control method for high-speed microjets.
\end{abstract}

This work was supported by JSPS KAKENHI Grant Numbers 26709007, 16J08521, 17H01246 and 17H02808.

Yoshiyuki Tagawa

tagawayo@cc.tuat.ac.jp

Keisuke Hayasaka

k_hayask@cc.tuat.ac.jp

Akihito Kiyama

kiyama@cc.tuat.ac.jp

1 Department of Mechanical Systems Engineering, Tokyo

University of Agriculture and Technology, Naka-cho

2-24-16, Koganei, Tokyo 184-8588, Japan
Keywords Microjet $\cdot$ Shockwave $\cdot$ Pressure impulse · Peak pressure

\section{Introduction}

The fundamental theory of a focused liquid jet induced by shockwaves has been investigated for many years (Birkhoff et al. 1948; Milgram 1969; Antkowiak et al. 2007; Kiyama et al. 2016). Recently, there is considerable interest in the jet inside a microchannel for use in microfluidic devices since microjets can be applied for biomedical applications (Pitt et al. 2004; Tagawa et al. 2013), printing technology (Delrot et al. 2016), the generation of nano-jet (Xiong et al. 2015), among others. The unique feature of the microjets is that the timescale of the jet formation (fluid dynamics timescale) $t_{\mathrm{f}}$ becomes closer to that of shockwave propagation (acoustic timescale) $t_{\mathrm{a}}$ compared with macroscale case. For instance, in macroscale case (e.g., Milgram (1969)), $t_{\mathrm{f}} \sim R / V \sim O(10) \mathrm{ms}$ ( $R$ : the curvature of the free surface $O(10) \mathrm{mm}, V$ : the typical flow velocity $O(1) \mathrm{m} / \mathrm{s}$ ) and $t_{\mathrm{a}} \sim L / c \sim O(0.01) \mathrm{ms}$ ( $L$ : the length of the liquid column $O(10) \mathrm{mm}, c$ : the speed of sound $O(1000) \mathrm{m} / \mathrm{s}$ ), meaning $t_{\mathrm{f}} / t_{\mathrm{a}} \sim 1000$. In contrast, in microjets case (the present research), $t_{\mathrm{f}} \sim R / V \sim O(10) \mu \mathrm{s}$ and $t_{\mathrm{a}} \sim L / c \sim O(1) \mu$ $\mathrm{s}$, meaning $t_{\mathrm{f}} / t_{\mathrm{a}} \sim 10$. It indicates that pressure profile of shockwaves might play a role on the jet in a microchannel. However, it has not been examined experimentally.

This study investigates the high-speed microjet induced by the shockwave propagation in a microchannel. We focus on the jet velocity, which is an essential quantity of microjets (Tagawa et al. 2012). For macroscale case, the velocity is supposed to be determined by a peak pressure value of shock front (Thompson 1972). For microscale case, however, Peters et al. (2013) had examined microjets using Boundary 
Integral simulation and indicated that summation of pressure fluctuation (i.e., pressure impulse) decides the velocity of the microjet. We elucidate dominant quantity for microjets velocity by varying separately the peak pressure and pressure impulse using non-spherical laser-induced shockwaves (Tagawa et al. 2016).

In addition, we discuss the cavitation upon a rarefaction wave reflected at the free surface since its onset is another factor for the jet velocity (Kiyama et al. 2016). The pressure inside a channel is relaxed when cavitation occurs (Daily et al. 2014), leading to jet velocity increment (Kiyama et al. 2016). It has been considered that the peak pressure of the shock front determines cavitation inception in macroscale (Caupin and Herbert 2006; Herbert et al. 2006). While for latter stage of cavitation dynamics, it is indicated that pressure impulse describes the bubble expansion (Ory et al. 2000). We investigate the dominant factor (the peak pressure or the pressure impulse) for cavitation inception in a microchannel.

These discussions should bring us insights for designing the microfluidic device using microjets.

\section{Method}

Two experimental setups were constructed for this study. The first setup was used to determine the effect of the shockwave pressure on the jet velocity in a microtube settled in a water tank (Fig. 1). The second setup was used to investigate the effect of the shockwave pressure on cavitation in an L-shaped microchannel (Fig. 2).

\subsection{Simultaneous measurement of underwater shockwave pressure and microjet velocity (water tank experiments)}

Figure 1 shows the setup for measuring the underwater shockwave pressure and microjet velocity (hereafter, "water tank experiments"). The method and conditions for generating shockwaves are the same as those used in our previous study (Tagawa et al. 2016). Underwater shockwaves are generated by a $532-\mathrm{nm}$ pulsed laser (Nd:YAG; Nano S PIV, Litron Lasers) with a pulse duration of $6 \mathrm{~ns}$, focused through an objective lens (MPLN series, Olympus) inside a tank $\left(300 \times 300 \times 450 \mathrm{~mm}^{3}\right)$ filled with water. The water is distilled using a water purification system (Milli-Q Integral, Merck; $13 \mathrm{M} \Omega \mathrm{cm})$ at room temperature $\left(15 \sim 20^{\circ} \mathrm{C}\right)$ and gas saturated. Using three types of objective lenses makes it possible to change the magnitudes of the peak pressure and pressure impulse of the shockwave. Table 1 shows the characteristics of each lens. The range of laser energy is $3-10 \mathrm{~mJ}$, and the measurement error is $\pm 5 \%$.
Table 1 Variety of the microscope objective

\begin{tabular}{lllll}
\hline Magnification & N.A & W.D. $(\mathrm{mm})$ & F.A. & Diameter $(\mu \mathrm{m})$ \\
\hline $5 \times$ & 0.10 & 20.0 & $1^{\circ}$ & 6.5 \\
$10 \times$ & 0.25 & 10.6 & $4^{\circ}$ & 2.6 \\
$20 \times$ & 0.25 & 25.0 & $6^{\circ}$ & 2.6 \\
\hline
\end{tabular}

N.A. the numerical aperture, W.D. the working distance, F.A. the focusing angle of the objective lens, Diameter the diameter of the focused laser beam

The experiment is carried out using two setups (Fig. 1a, b) in order to obtain the shockwave pressure and jet velocity in two directions. In Fig. 1a, hydrophone (Needle Probe, Mueller Instruments; measurable range: -10 to $100 \mathrm{MPa}$; rise time: $50 \mathrm{~ns}$, measurement error: $\pm 2 \%$ (Zhou et al. 2016)) is installed along the laser irradiation direction (hereafter, $\left.\theta=0^{\circ}\right)$, and a narrow glass tube $(d=500 \mu$ $\mathrm{m})$ is installed perpendicular to the laser irradiation direction $\left(\theta=90^{\circ}\right)$. Note that $\theta$ denotes the angle from the laser irradiation direction. In Fig. 1b, the narrow glass tube is installed along the laser irradiation direction $\left(\theta=0^{\circ}\right)$, and the hydrophone is installed perpendicular to the laser irradiation direction $\left(\theta=90^{\circ}\right)$. The time history of the shockwave pressure is measured using an oscilloscope (ViewGo II DS5554A, IWATSU; rise time: $750 \mathrm{ps;} \mathrm{maximum} \mathrm{sampling}$ rate: $2 \mathrm{GS} / \mathrm{s}$ ) connected to the hydrophone. The distance from the laser focal point to the end faces of the hydrophone and narrow glass tube is $5.0 \pm 0.1 \mathrm{~mm}$. One end of the narrow glass tube is connected via a plastic tube to an air-filled syringe. The position of the air-liquid interface in the glass tube is adjusted using a syringe pump (ULTRA 70-3005, Harvard). The other end of the glass tube is open. The position of the air-liquid interface is adjusted to be at the end of the tube. When a laser-induced shockwave arrives at the air-liquid interface, the air-liquid interface forms a jet directed toward the inside of the narrow tube. The jet velocity $V_{\mathrm{j}}$ is measured from images captured using a high-speed camera (FASTCAM SA-X, Photron) with a spatial resolution of $1024 \times 124$ pixels and a recording rate of $80,000 \mathrm{fps}$. Specifically, $V_{\mathrm{j}}$ is measured between the image taken at the instant that the air-liquid interface inside the glass tube focuses and the next image, using the distance travelled by the tip of the jet. An LED light source (KL 1600 LED, OLYMPUS) is used. The pulsed laser and high-speed camera are synchronized using a delay generator (Model 575 Pulse/Delay Generator, BNC).

\subsection{Microjet formation in $\mathrm{L}$-shaped microchannel (L-shaped microchannel experiments)}

Figure 2 shows the setup for forming a microjet inside the narrow tube (hereafter, "L-shaped microchannel 
Fig. 1 Experimental setup for measuring shock pressure and jet velocity (water tank experiments). Expanded views show the measurement area in the water tank. The laser spot is situated on the origin. a The hydrophone is placed on $\theta=0^{\circ}$ $(y$-axis) and the glass tube on $\theta=90^{\circ}$ ( $x$-axis). b The hydrophone on $\theta=90^{\circ}$ ( $x$-axis) and a glass tube on the $\theta=0^{\circ}$ (y-axis)

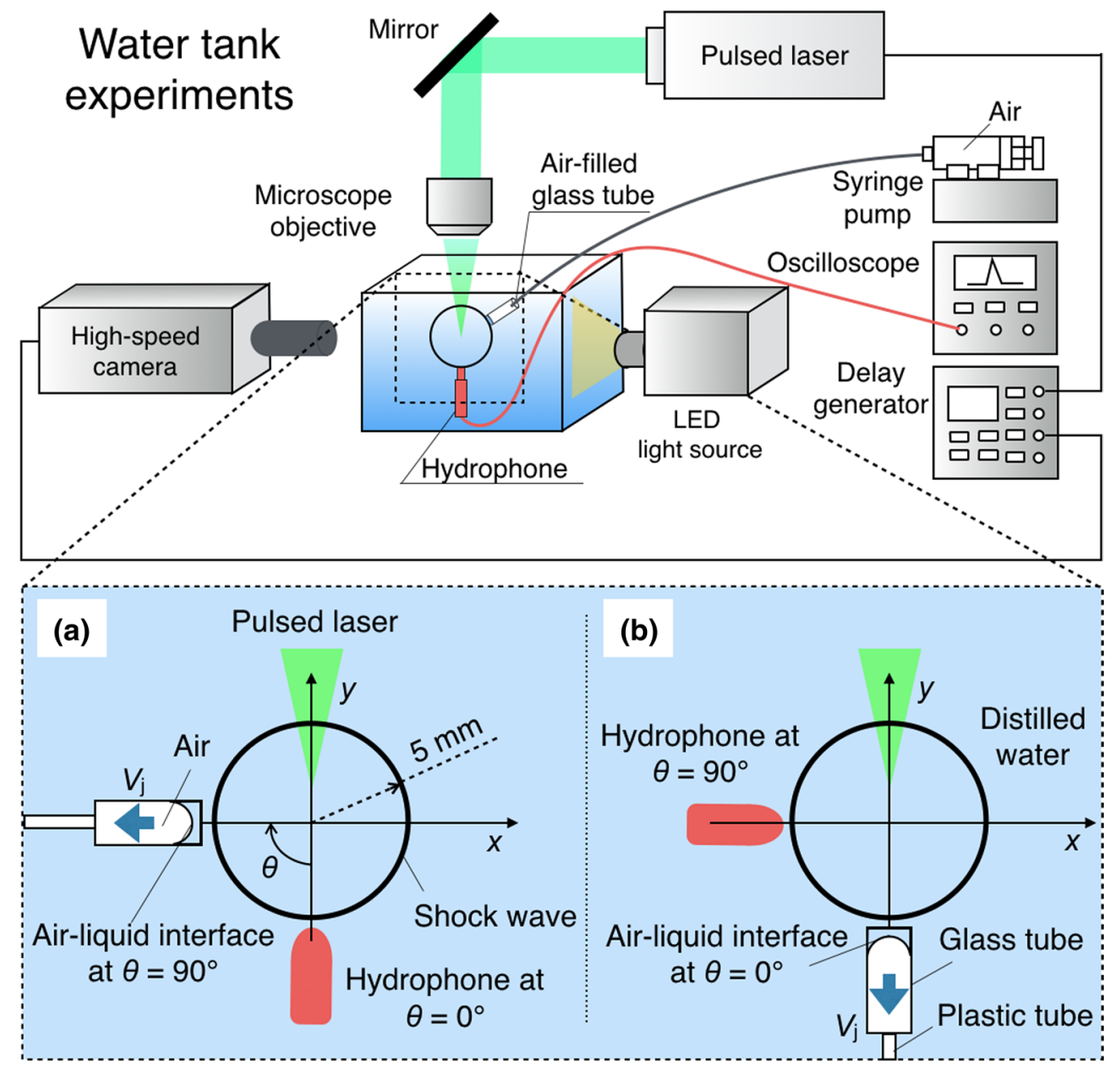

experiments"). The equipment used to generate the jet and measure its velocity is the same as for the experiments in the water tank. The jet is formed inside the L-shaped microchannel (quartz glass, $10 \times 5 \times 25 \mathrm{~mm}^{3}$ ). One section of the microchannel ( $800 \mu \mathrm{m}$ in diameter) is connected to a syringe filled with dyed water. The energy absorption efficiency of dyed water is higher than that of pure water. Therefore, laserinduced bubbles can occur at lower laser energies than in the water tank experiments. The range of input energy of the laser is $102-625 \mu \mathrm{J}$, and the measurement error is $\pm 10 \%$. Only the $10 \times$ objective lens is used. The position of the air-liquid interface is manipulated using a syringe pump. The range of distance $H$ from the air-liquid interface to the laser-induced bubble is $800-2700 \mu \mathrm{m}$. The other section of the microchannel (diameter $d=500 \mu \mathrm{m}$ ) is open to the atmosphere and contains the air-liquid interface. The pulsed laser is focused on the orthogonal portion of the microchannel. In Fig. 2a, the air-liquid interface that generates the jet is perpendicular to the laser irradiation direction $\left(\theta=90^{\circ}\right)$. In Fig. 2b, the air-liquid interface is in the laser irradiation direction $\left(\theta=0^{\circ}\right)$. Because the inside diameter of the microchannel is much smaller than the radius of the hydrophone, it is not possible to measure the pressure. In this experiment, the pressure distribution of the underwater shockwave generated is assumed to be the same as the pressure distribution of the shockwave generated in the water tank experiments.

\section{Results and discussion(water tank experiments)}

\subsection{Preliminary experiment}

This section confirms that the shockwaves used in this study are essentially the same as that in our previous report (Tagawa et al. 2016). Figure 3 shows time histories of shockwave pressure in the two directions measured in the water tank experiments. Note that the pressure in each direction is not measured simultaneously. The horizontal axis shows elapsed time from laser irradiation, and the vertical axis shows pressure in gauge. The laser energy was taken to be $3.0 \pm 0.6 \mathrm{~mJ}$. The pressure waveform obtained for $\theta=90^{\circ}$ differs greatly from that obtained for $\theta=0^{\circ}$. For $\theta=90^{\circ}$, there is one sharp pressure peak. For $\theta=0^{\circ}$, the pressure changes gradually, and there is a greater number of pressure peaks. This agrees well with our report (Tagawa et al. 2016), so the shockwave generated using 
Fig. 2 Experimental setup for generating microjets in L-shaped microchannel (L-shaped microchannel experiments). $\mathbf{a}$ and $\mathbf{b}$ Correspond to the microjet directed toward $\theta=90^{\circ}$ ( $x$-axis) and $\theta=0^{\circ}$ ( $y$-axis), respectively. The laser is incident from the positive side of the $y$-axis

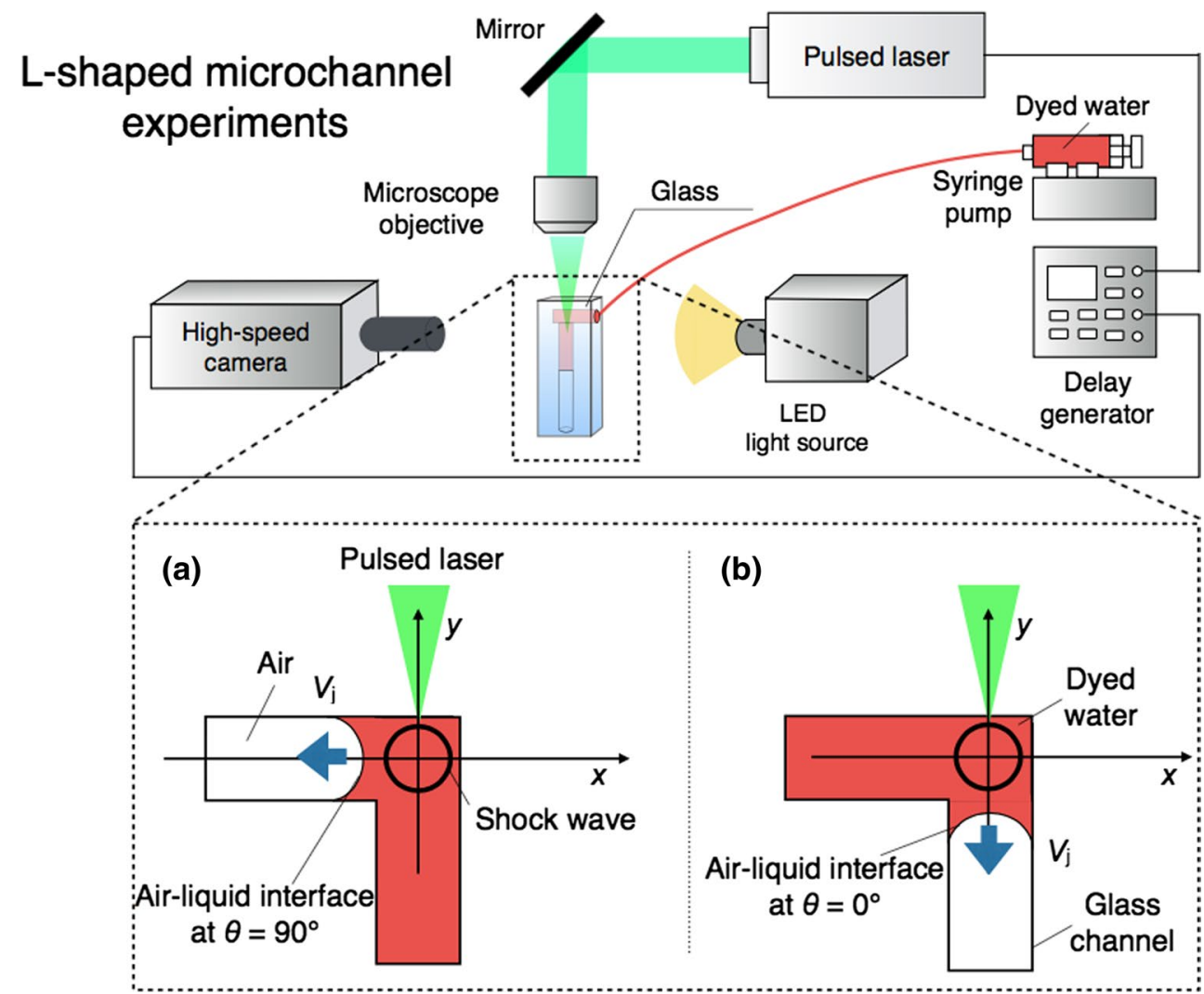

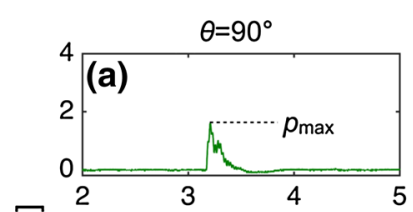
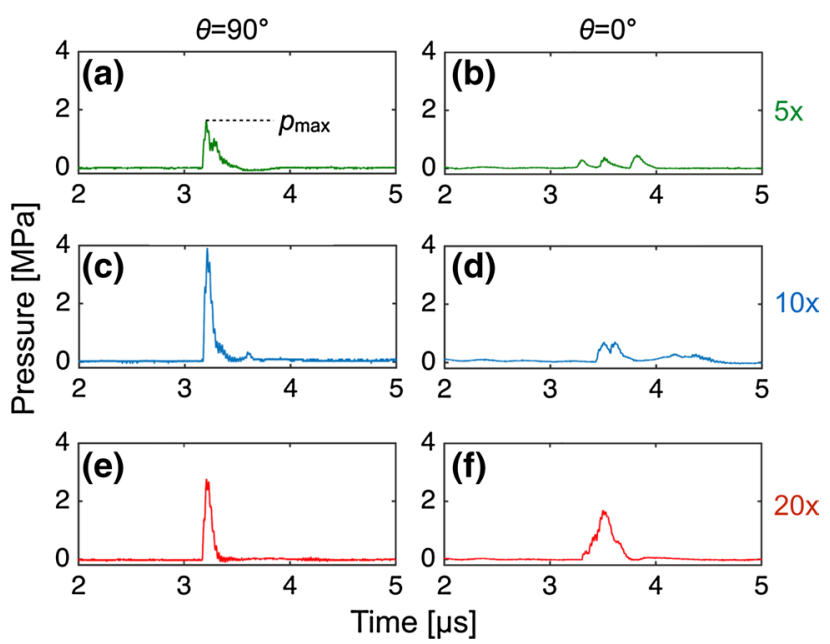

Fig. 3 Pressure versus time measured by hydrophones placed at $\theta=90^{\circ}$ and $\theta=0^{\circ}$. The horizontal and vertical axes represent the time from laser irradiation and the pressure detected by the hydrophone, respectively. a Hydrophone: $\theta=90^{\circ}$, magnification: $5 \times$; b hydrophone: $\theta=0^{\circ}$, magnification: $5 \times$; $\mathbf{c}$ hydrophone: $\theta=90^{\circ}$, magnification: $10 \times$; d hydrophone: $\theta=0^{\circ}$, magnification: $10 \times$; e hydrophone: $\theta=90^{\circ}$, magnification: $20 \times$; $\mathbf{f}$ hydrophone: $\theta=0^{\circ}$, magnification: $20 \times$. The laser energy is $3.0 \mathrm{~mJ}$

these experimental setups is considered to have an nonspherically-symmetric pressure distribution, similar to that generated previously. In our report (Tagawa et al. 2016), we discussed the mechanism in detail and demonstrated that the peak pressure of the shockwave differs according to the propagation direction, but the pressure impulse is equal regardless of the propagation direction. Thus, in this paper, we assumed that the pressure impulse for both propagation directions are equal.

\subsection{Effect of pressure impulse and peak pressure on microjet velocity}

The pressure impulse is given by the following equation:

$I=\int p \mathrm{~d} t$

Here, $I, t$, and $p$ are, respectively, indicate the pressure impulse, the time, and the pressure. Figure 4 shows the jet velocity versus the pressure impulse, where the range of laser energy is $3-10 \mathrm{~mJ}$. The horizontal axis shows the pressure impulse obtained using the hydrophone, and the vertical axis shows the velocity of the jet generated in the tube. The plots represent mean values obtained after conducting the test five times, and the error bars indicate the corresponding standard deviations. When the pressure impulse is below $0.2 \mathrm{~Pa} \mathrm{~s}$, the jet velocity is zero. When the pressure impulse is above $0.2 \mathrm{~Pa} \mathrm{~s}$, the jet velocity is roughly proportional to 


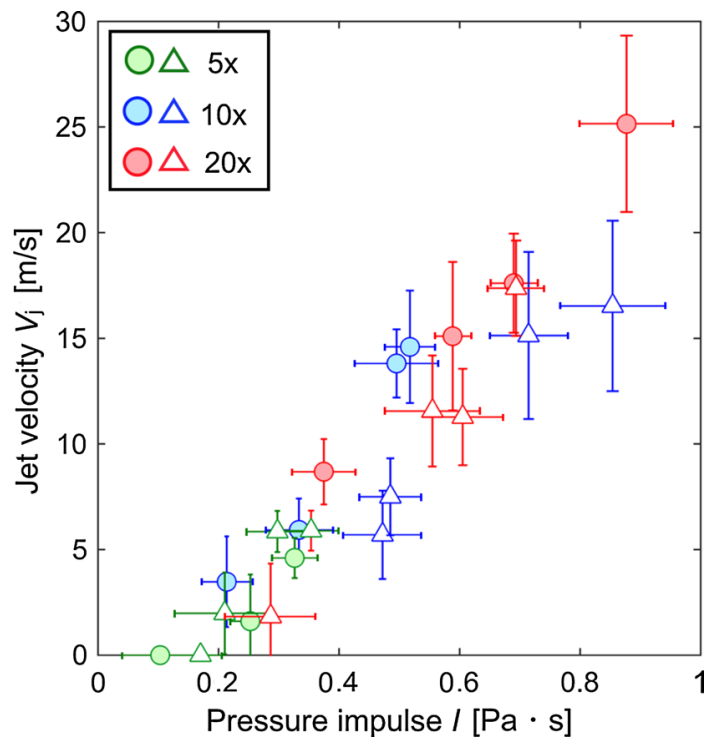

Fig. 4 Microjet velocity versus pressure impulse. Circle markers: the glass tube and the hydrophone are arranged on $\theta=0^{\circ}$ and $\theta=90^{\circ}$, respectively (see Fig. 2a). Triangle markers: the glass tube and the hydrophone are arranged on $\theta=90^{\circ}$ and $\theta=0^{\circ}$, respectively (see Fig. 2b). Green, blue and red plots designate experiments with a $5 \times$ , $10 \times, 20 \times$ objective microscope lens, respectively. The error bars correspond to the standard deviation calculated from 5 trials (color figure online)

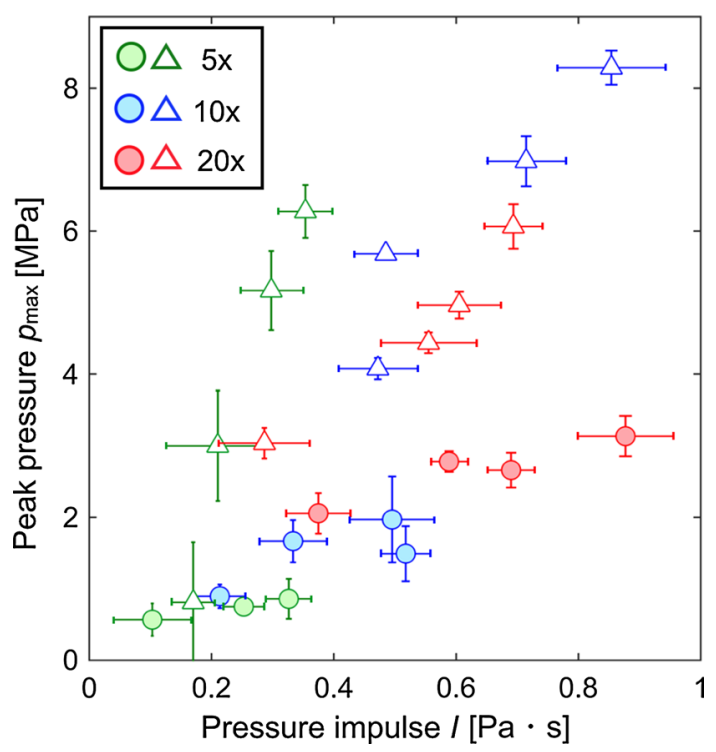

Fig. 5 Peak pressure versus pressure impulse. Markers and colors mean the same as shown in Fig. 4 (color figure online)

the pressure impulse. In addition, the jet velocity is almost constant regardless of the type of objective lens and the orientation of the equipment.

Figure 5 shows the peak pressure versus the pressure impulse measured using the hydrophone. Both quantities are measured simultaneously using one hydrophone. The peak pressure is proportional to the pressure impulse, but the gradient strongly depends on the propagation direction. The peak pressure for $\theta=90^{\circ}$ is approximately three times higher than that for $\theta=0^{\circ}$. If the jet velocity was dependent on the peak pressure, the jet velocities in Fig. 4 would vary according to the direction of propagation. However, since this is not the case, the jet velocity is considered to be dependent on the pressure impulse, rather than the peak pressure. The timescale for jet formation $t_{\mathrm{f}} \sim R / V_{\mathrm{j}}$ is approximately $25 \mu$ $\mathrm{s}\left(R \sim 250 \mu \mathrm{s}, V_{\mathrm{j}} \approx 10 \mathrm{~m} / \mathrm{s}\right)$, which is much larger than pressure duration of the shockwave (typically less than $2 \mu \mathrm{s}$, see Fig. 3). Therefore the entire pressure impulse contributes to jet formation and determines microjet velocity. Note that the Laplace pressure $(p=2 \sigma / R)$ on free surface is approximately $0.3 \mathrm{kPa}$ (surface tension $\sigma \sim 70 \mathrm{mN} / \mathrm{m}, R \sim 250 \mu \mathrm{m}$ ), which is much lower than the shock pressure $(\sim O(1) \mathrm{MPa})$. It indicates that the surface tension does not hinder the jet formation for both $\theta=0^{\circ}$ and $\theta=90^{\circ}$.

\section{Results and discussion (L-shaped microchannel experiments)}

\subsection{Changes in microjet velocity with presence/absence of cavitation}

In the water tank experiments, when the distance from the laser-induced bubble to the air-liquid interface was $5 \mathrm{~mm}$, the lowest peak pressure of the underwater shockwave was approximately $0.5 \mathrm{MPa}$ (Fig. 5). If the shockwave traveling inside the L-shaped microchannel is comparable to that in the water tank, the rarefaction wave reflecting at the air-liquid interface inside the L-shaped microchannel is considered to be smaller than the saturated vapor pressure of water $(\approx 2 \mathrm{kPa})$. Therefore, the rarefaction wave is considered to cause cavitation in the liquid.

Kiyama et al. (2016) conducted macroscales experiments and reported that the onset of cavitation can change the jet velocity. The controllability of microjet velocity may get worse if the similar mechanism holds in a microchannel as well. Thus, here we address this issue. First, we look at microjets with lower energy (absence of cavitation). The jet velocity is not subject to the action of cavitation, so it is expected to depend on the magnitude of pressure impulse and not to change with the laser irradiation direction. Figure 6 a shows snapshots taken during microjet formation for $\theta=0^{\circ}$. In the initial state $(t=0)$, the air-liquid interface has a concave shape. A laser-induced bubble occurs $(t=17.5 \mu$ $s)$ at the wall through which the laser passes. The air-liquid interface displaces immediately, and a focused microjet forms. Figure $6 \mathrm{~b}$ shows the jet velocity $V_{\mathrm{j}}$ for various values of $H$, in the case of $\theta=0^{\circ}$ and $\theta=90^{\circ}$. The vertical and 
Fig. 6 a Photograph of the microjet generated during experiments in the L-shaped microchannel for $\theta=0^{\circ} . H=$ $1080 \mu \mathrm{m}$. The laser is incident from the right side, and the laser energy is $185 \mu \mathrm{J}$. b Jet velocity as a function of distance from laser-induced bubble to the air-liquid interface. The laser is incident from the right side, and the laser energy is $185 \mu \mathrm{J}$. The circles and triangles designate results for $\theta=0^{\circ}$ and $\theta=90^{\circ}$, respectively. The error bars are the standard deviation
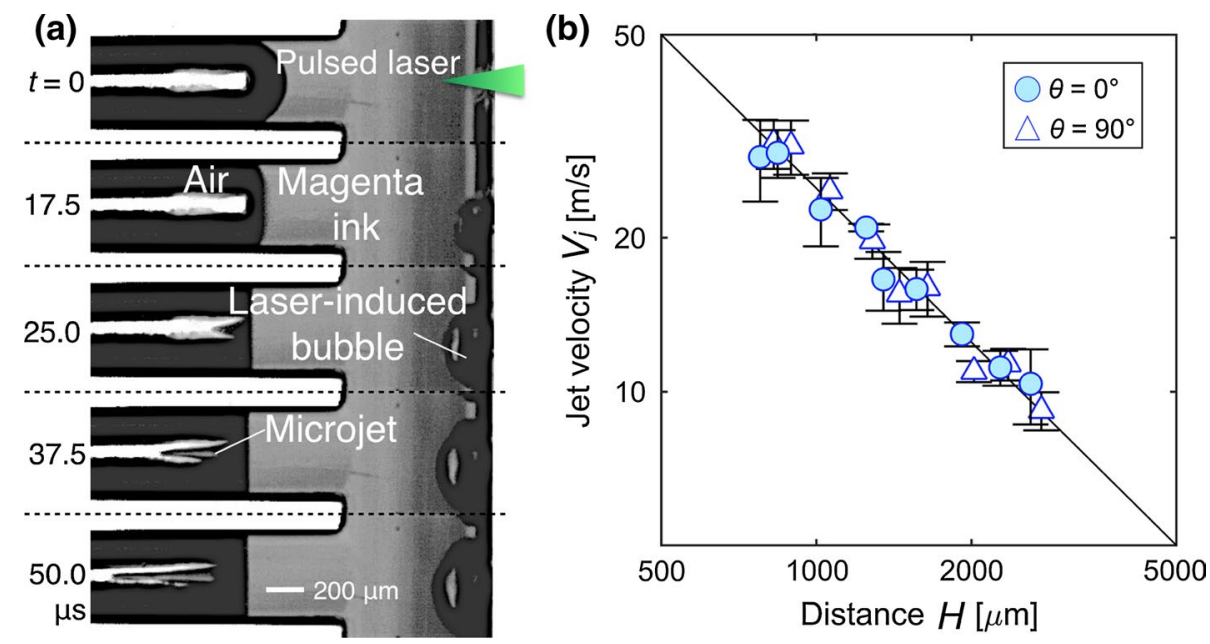

horizontal axes are logarithmic. The laser energy is constant at $185 \mu \mathrm{J}$. The plots represent mean values obtained after conducting the test five times, and the error bars indicate the standard deviations. The solid line shows the gradient of the inversely proportional relationship (Tagawa et al. 2012; Peters et al. 2013). Note that, for $\theta=0^{\circ}$, the contact angle between the air and the liquid at the air-liquid interface changes with $H$ due to the effect of gravity, so the values of $V_{\mathrm{j}}$ take account of changes in the contact angle (Tagawa et al. 2012). All data collapse into one trend (black solid line) so the jet velocity for $\theta=0^{\circ}$ is approximately equal to that for $\theta=90^{\circ}$. The pressure impulse acting on the air-liquid interface was equal in each setup, so the jet velocity is considered to be roughly constant regardless of direction, as expected. Figure $6 \mathrm{~b}$ also suggests that the relationship between direction and the pressure profile in dyed water has similar trend to that in pure water, even if generation processes of shockwave in both liquids might be different [i.e., vaporization in dyed water and plasma-mediated ablation in pure water, see Vogel and Venugopalan (2003)].

Next, we look at microjets with higher energy. Figure $7 \mathrm{a}$ shows snapshots taken during microjet formation for $\theta=0^{\circ}$.
The distance $H$ is $2000 \mu \mathrm{m}$. When $t=17.5 \mu \mathrm{s}$, cavitation occurs near the air-liquid interface, which is thought to have been generated by the rarefaction wave reflected at the air-liquid interface. Figure $7 b$ shows the relationship between $H$ and $V_{\mathrm{j}}$. The laser energy is constant at $650 \mu \mathrm{J}$. Cavitation occurred in most cases when the microjets measured in Fig. 7 formed. For $\theta=0^{\circ}$, the relationship between $H$ and $V_{\mathrm{j}}$ is inversely proportional (see black solid line), and this agrees well with that in Fig. 6a. On the other hand, for $\theta=90^{\circ}$, the inversely proportional relationship no longer holds when $H>1500$.

\subsection{Probability of cavitation}

We investigated that the difference of cavitation probability to understand the jet velocity increment for larger $H$ for $\theta=90^{\circ}$. Figure 8 shows the probability of cavitation occurring in the L-shaped microchannel. The horizontal axis is the distance $H$ from the laser-induced bubble to the air-liquid interface, and the vertical axis is the probability of cavitation. Each plot represents the probability from five trials. In this case, the laser energy is constant at $650 \mu \mathrm{J}$.
Fig. 7 a Photograph of the microjet generated at $\theta=0^{\circ}$ with the cavitation bubbles. $H=2000 \mu \mathrm{m}$. The laser is incident from the right side, and the laser energy is $650 \mu \mathrm{J}$. b Jet velocity as a function of distance from laser-induced bubble to the air-liquid interface. The laser is incident from the right side, and the laser energy is $650 \mu \mathrm{J}$. The circles and triangles designate results for $\theta=0^{\circ}$ and $\theta=90^{\circ}$, respectively. The error bars are the standard deviation (a)

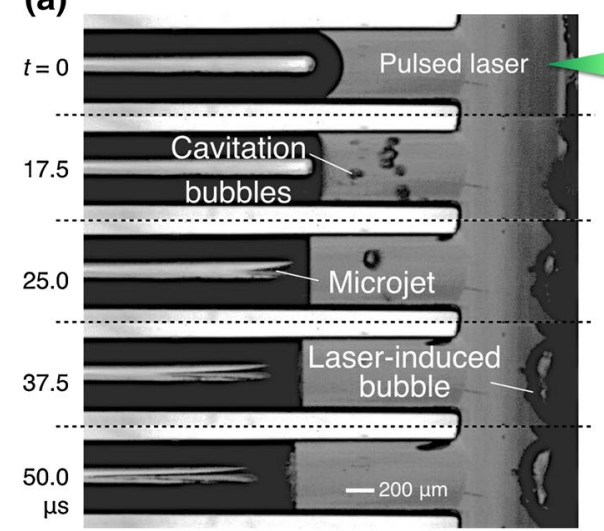

(b)

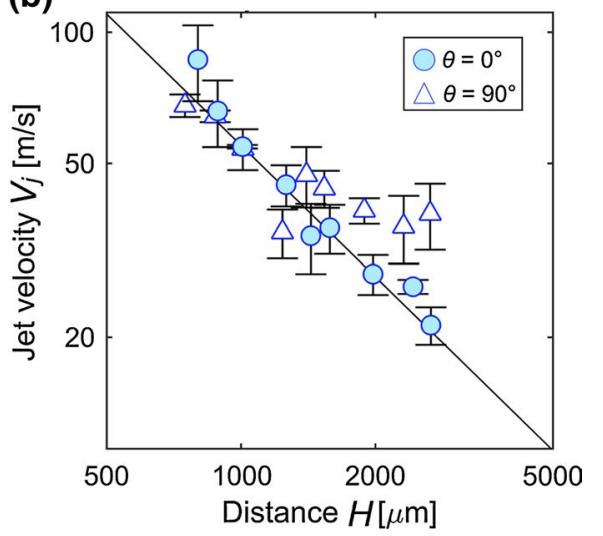




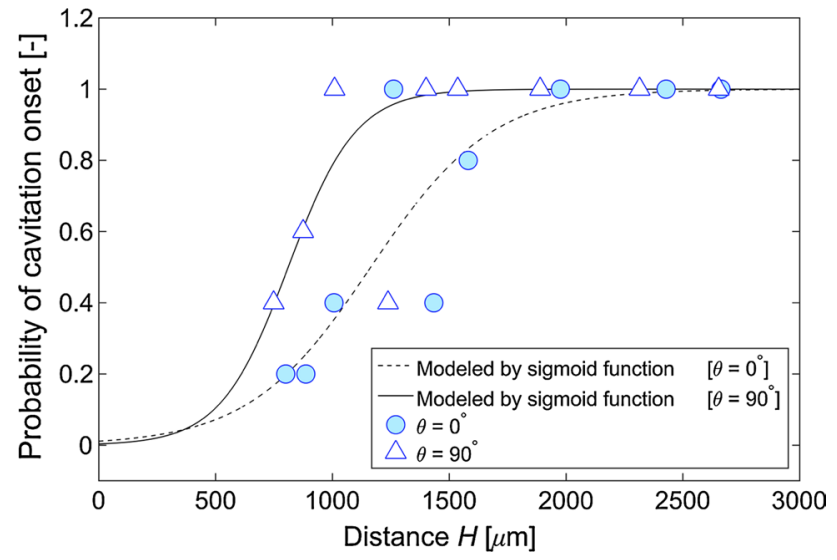

Fig. 8 Probability of cavitation as a function of distance from a laserinduced bubble to meniscus. The circles and triangles are the probabilities obtained for $\theta=0^{\circ}$ and $\theta=90^{\circ}$, respectively. The black line and dotted line are fits using a sigmoid function

As $H$ increases, the amount of bubble nuclei is considered to increase with the volume of fluid. Therefore, the probability of cavitation increases with $H$ (Kiyama et al. 2016). The probability of cavitation is approximated using the least-squares method using the sigmoid function [Prob. $=1$ $\left.\cdot[1+\exp \langle-\{(x-a) / b\}\rangle]^{-1}\right]$ (Maxwell et al. 2011). The solid line $(a=811, b=145)$ indicates the approximate curve for $\theta=90^{\circ}$, and the dotted line $(a=1164, b=260)$ indicates the approximate curve for $\theta=0^{\circ}$. When $H>2000$, the probability of cavitation at both directions is 1 . Interestingly, when $H \leq 2000$, the probability at $\theta=90^{\circ}$ is greater than at $\theta=0^{\circ}$.

Cavitation can occur when the pressure around the bubble nucleus is lower than the vapor pressure. A large negative pressure increases the probability of cavitation (Maxwell et al. 2011). The magnitude of the negative pressure is considered to be proportional to the magnitude of the shockwave before it is reflected at the air-liquid interface (Fig. 3). Therefore, the magnitude of the peak value of negative pressure at $\theta=90^{\circ}$ is considered to be greater than that at $\theta=0^{\circ}$. In fact, as already mentioned, the peak pressure of shockwave for $\theta=90^{\circ}$ is higher than that for $\theta=0^{\circ}$. The above results suggest that the probability of cavitation is affected by the magnitude of the peak pressure of the shockwave.

Here we discuss a possible scenario based on the theory of bubble dynamics. The lifetime of cavitation bubble (Delale 2012) in this study is approximate 20-30 $\mu$ s, much larger than pressure duration $(\sim O(1) \mu \mathrm{s})$. Nevertheless, pressure impulse does not play any dominant role. It is likely that the force balance in rapid expansion of a bubble is important. The motion of a homogeneous bubble is described by the Rayleigh-Plesset equation (Duguè et al. 1992). This equation indicates that the expansion of a small bubble (i.e., cavitation nucleus) requires large pressure difference surpassing the Laplace pressure $\left(=2 \sigma / R_{\mathrm{b}}\right.$, where $R_{\mathrm{b}}$ is the radius of a bubble) across the bubble surface. Unless the pressure difference exceeds the threshold (Laplace pressure $\sim O(1) \mathrm{MPa}$ ), the bubbles do not expand. Thus, the pressure impulse should not be considered as the criterion of cavitation onset.

Above findings might contribute to improve controllability of microjet velocity. When $H$ is relatively small, cavitation does not occur in a microchannel. Thus, the jet velocity is constant irrespective to the direction of a laser irradiation, leading to the increase in freedom for designing microjet generators. When $H$ and the laser energy are relatively large, cavitation occurs. Although it can cause the cavitation-assisted increment of microjet velocity, the controllability of jet velocity gets worse. Our experimental results (see Fig. 8) indicate that a suitable choice of the direction of laser irradiation reduces cavitation onset, leading to high controllability of microjet velocity.

\section{Conclusion}

In this paper, we experimentally investigate the effect of a shockwave on the velocity of a microjet and underwater cavitation onset in a microchannel, focusing on the pressure impulse and peak pressure of the shockwave. we conduct two kind of experiments using a microtube in a tank and an L-shaped microchannel. Using the first experimental setup, we investigated the effect of the shockwave pressure on the jet velocity in a water tank. We demonstrated that the jet velocity is dependent on the magnitude of the pressure impulse, regardless of the magnitude of the peak pressure. Using the second experimental setup, we investigated the effect of the shockwave pressure on cavitation in an L-shaped microchannel. We showed that the probability of cavitation changes according to the magnitude of the peak pressure. The jet velocity at the onset of cavitation can be considered to be affected by the peak pressure. In addition we discuss controllability of microjet velocity in a microchannel. We suggest that our novel findings have potentials to improve flexibility of the design or controllability of microjet velocity.

Open Access This article is distributed under the terms of the Creative Commons Attribution 4.0 International License (http://creativecommons.org/licenses/by/4.0/), which permits unrestricted use, distribution, and reproduction in any medium, provided you give appropriate credit to the original author(s) and the source, provide a link to the Creative Commons license, and indicate if changes were made. 


\section{References}

Antkowiak A, Bremond N, Le Dizès S, Villermaux E (2007) Shortterm dynamics of a density interface following an impact. J Fluid Mech 577:241-250

Birkhoff G, MacDougall DP, Pugh EM (1948) Explosives with lined cavities. J Appl Phys 19(6):563-582

Caupin F, Herbert E (2006) Cavitation in water: a review. C R Phys 7(9-10):1000-1017

Daily J, Pendlebury J, Langley K, Hurd R, Thomson S, Truscott T (2014) Catastrophic cracking courtesy of quiescent cavitation. Phys Fluids 26(9):091107

Delale CF (Ed) (2012) Bubble dynamics and shock waves. vol 8. Springer

Delrot P, Modestino MA, Gallaire F, Psaltis D, Moser C (2016) Inkjet printing of viscous monodisperse microdroplets by laser-induced flow focusing. Phys Rev Appl 6(2):024003

Duguè C, Fruman DH, Billard J-Y, Cerrutti P (1992) Dynamic criterion for cavitation of bubbles. Trans ASME J Fluids Eng 114(2):250-254

Herbert E, Balibar S, Caupin F (2006) Cavitation pressure in water. Phys Rev E 74(4):041603-22

Kiyama A, Tagawa Y, Ando K, Kameda M (2016) Effects of a water hammer and cavitation on jet formation in a test tube. J Fluid Mech 787:224-236

Maxwell AD, Wang T, Cain CA, Fowlkes JB, Sapozhnikov OA, Bailey MR, Xu Z (2011) Cavitation clouds created by shock scattering from bubbles during histotripsy. J Acoust Soc Am 130(4):1888-1898

Milgram JH (1969) The motion of a fluid in a cylindrical container with a free surface following vertical impact. J Fluid Mech 37(3):435-448
Ory E, Yuan H, Prosperetti A, Popinet S (2000) Growth and collapse of a vapor bubble in a narrow tube. Phys Fluids 12(6):1268-1277

Peters IR, Tagawa Y, Oudalov N, Sun C, Prosperetti A, Lohse D, van der Meer D (2013) Highly focused supersonic microjets: numerical simulations. J Fluid Mech 719:587-605

Pitt WG, Husseini GA, Staples BJ (2004) Ultrasonic drug delivery-a general review. Expert opin drug deliv 1(1):37-56

Tagawa Y, Oudalov N, Visser CW, Peters IR, van der Meer D, Sun C, Prosperetti A, Lohse D (2012) Highly focused supersonic microjets. Phys Rev X 2(3):031002

Tagawa Y, Oudalov N, El Ghalbzouri A, Sun C, Lohse D (2013) Needle-free injection into skin and soft matter with highly focused microjets. Lab Chip 13(7):1357-1363

Tagawa Y, Yamamoto S, Hayasaka K, Kameda M (2016) On pressure impulse of a laser-induced underwater shock wave. J Fluid Mech 808:5-18

Thompson PA (1972) Compressible fluid-dynamics. McGraw-Hill, USA

Vogel A, Venugopalan V (2003) Mechanisms of pulsed laser ablation of biological tissues. Chem Rev 103(2):577-644

Xiong S, Chin LK, Ando K, Tandiono T, Liu AQ, Ohl CD (2015) Droplet generation via a single bubble transformation in a nanofluidic channel. Lab Chip 15:1451-1457

Zhou H, Zhang Y, Han R, Jing Y, Wu J, Liu Q, Ding W, Qiu A (2016) Signal analysis and waveform reconstruction of shock waves generated by under-water electrical wire explosions with piezoelectric pressure probes. Sensors 16(4):573 